\title{
Electronic viscosity in a quantum well: A test for the local-density approximation
}

\author{
Roberto D'Agosta* and Massimiliano Di Ventra \\ University of California-San Diego, La Jolla, California 92093, USA \\ Giovanni Vignale \\ University of Missouri-Columbia, Columbia, Missouri 65211, USA
}

(Received 22 February 2007; published 17 July 2007)

\begin{abstract}
In the local-density approximation (LDA) for electronic time-dependent current-density-functional theory, many-body effects are described in terms of the viscoelastic constants of the homogeneous three-dimensional electron gas. In this paper, we critically examine the applicability of the three-dimensional LDA to the calculation of the viscous damping of one-dimensional collective oscillations of angular frequency $\omega$ in a quasitwo-dimensional quantum well. We calculate the effective viscosity $\zeta(\omega)$ from perturbation theory in the screened Coulomb interaction and compare it with the commonly used three-dimensional LDA viscosity $Y(\omega)$. Significant differences are found. At low frequency, $Y(\omega)$ is dominated by a shear term, which is absent in $\zeta(\omega)$. At high frequency, $\zeta(\omega)$ and $Y(\omega)$ exhibit different power-law behaviors $\left(\omega^{-3}\right.$ and $\omega^{-5 / 2}$, respectively), reflecting different spectral densities of electron-hole excitations in two and three dimensions. These findings demonstrate the need for better approximations for the exchange-correlation stress tensor in specific systems where the use of the three-dimensional functionals may lead to unphysical results.
\end{abstract}

DOI: 10.1103/PhysRevB.76.035320

PACS number(s): 73.21.Fg, 73.22.Lp, 73.20.-r, 71.15.Mb

\section{INTRODUCTION}

Time-dependent density-functional theory is one of the premier techniques for the study of the dynamics of quantum many-body systems. ${ }^{1-4}$ The central idea of the theory, dating back to the pioneering work of Kohn and co-workers, ${ }^{5,6}$ is that the interacting many-body system can be simulated by a noninteracting system that yields the same density under the action of the self-consistent electrostatic potential $V_{H}$ and an additional exchange-correlation $(\mathrm{xc})$ potential $V_{x c}$ that is uniquely determined by the particle density $n .^{7}$ This approach, while rigorous in principle, runs into serious difficulties when applied to phenomena that involve dissipation and memory, for example, the damping of collective modes. This is because $V_{x c}$ is a strongly nonlocal functional of the density. ${ }^{8}$

About 10 years ago, a time-dependent current-densityfunctional theory (TDCDFT) was proposed,,${ }^{9,10}$ which offered a natural way of treating memory and dissipation without losing the advantages of a local description of manybody effects. In this theory the exchange-correlation potential was replaced by an exchange-correlation force field $\vec{F}_{x c}=-e \vec{E}_{x c}$ (the notation is designed for electronic systems: $-e$ is the electron charge and $\vec{E}_{x c}$ is an "exchange-correlation electric field") which was represented as the divergence of a

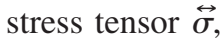

$$
\vec{F}_{x c}=-e \vec{E}_{x c}=\frac{1}{n} \vec{\nabla} \cdot \overleftrightarrow{\sigma}
$$

which in turn was expressed as a local functional of the equilibrium density $n$ and the velocity $\vec{v}=\frac{\vec{j}}{n}$, where $\vec{j}$ is the particle current density. Recently, TDCDFT has been extended to open quantum systems by two (Di Ventra and R. D'Agosta) of the present authors. ${ }^{11}$
In the linear-response regime, an approximate form of the stress tensor was proposed, ${ }^{8,10}$ which has the same form as the stress tensor of the classical Navier-Stokes hydrodynamics: ${ }^{12}$

$$
\sigma_{x c, i j}=-p_{x c} \delta_{i j}+\eta\left(\frac{\partial v_{i}}{\partial r_{j}}+\frac{\partial v_{j}}{\partial r_{i}}-\frac{2}{3} \vec{\nabla} \cdot \vec{v} \delta_{i j}\right)+\zeta \vec{\nabla} \cdot \vec{v} \delta_{i j},
$$

where $p_{x c}$ is the exchange-correlation pressure of the homogeneous electron gas at the local density $n, \eta$ is the shear viscosity, and $\zeta$ is the bulk viscosity of the homogeneous electron gas at the local equilibrium density $n_{0} . \eta$ and $\zeta$ are frequency dependent and have imaginary parts which are related to a shear modulus, and a dynamical bulk modulus respectively.

An essential feature of this approximation is that it is still local in the equilibrium density and the velocity field. In particular, the adiabatic local-density approximation (LDA) [or three-dimensional LDA (3D-LDA)] amounts to keeping only the first term on the right-hand side of Eq. (2).

The exchange-correlation field (1) with stress tensor (2) has been applied to the study of several systems (semiconductor quantum wells, ${ }^{13-15}$ atoms, ${ }^{16}$ semiconductors and polymers, ${ }^{17-19}$ molecular junctions, ${ }^{12,20-22}$ and metals ${ }^{23,24}$ ) with varying degrees of success. However, a fundamental difficulty exists. Because the viscosities $\eta$ and $\zeta$ are borrowed from an infinite three-dimensional electron gas, this approximation implicitly assumes the existence of a continuum spectrum of excitations (electron-hole pairs). Needless to say, this assumption is not justified in systems with discrete energy levels. This may lead in some cases to spurious results. For example, the optical transitions between discrete energy levels of atoms are found to have a spurious linewidth. ${ }^{16}$ 


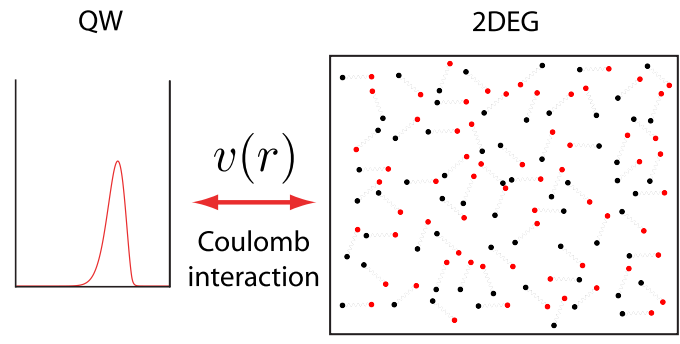

FIG. 1. (Color online) Two-subsystem model for electrons in a two-dimensional quantum well. The collective motion of the electrons in the $z$ direction is coupled, through the Coulomb interaction, to density fluctuations in the plane of the 2DEG. Energy is exchanged between the two subsystems via the creation of electronhole pairs in the 2DEG.

A more "friendly" system is the electron gas in a twodimensional quantum well (shown in Fig. 1), in which the electrons perform collective oscillations in a direction perpendicular to the plane of the quantum well (the $z$ direction), while remaining homogeneous in the plane of the well (the $x-y$ plane). Spontaneous oscillations of this kind-known as intersubband plasmons - have been intensively studied because of their possible use in devices in the terahertz frequency range. In practice, these oscillations, spontaneous or forced, provide one of the best testing grounds for TDDFT calculations of damping and relaxation. ${ }^{16,25,26}$ Even in this favorable case, however, one may question the accuracy of the local-density approximation for the viscosity. To begin with, the electron-hole pairs that are responsible for the damping and the screening of the electron-electron interaction should be those of an essentially two-dimensional electron gas (2DEG) - not those of a three-dimensional electron gas as implicitly assumed in the 3D-LDA. However, the system is not exactly two dimensional, even if one neglects intersubband transitions, because the electron-electron interaction in the lowest subband is modified by form factors, which take into account the finite extent of the wave function in the $z$ direction. More importantly, in a collective oscillation that preserves uniformity in the $x-y$ plane, the Fermi surface of the 2DEG does not change its (circular) shape (see
Fig. 2): this means that only the bulk viscosity $\zeta$ contributes to the damping of the collective mode. By contrast, the dominant contribution to the damping in 3D-LDA comes from the shear viscosity, which is associated with changes in shape of the implicitly assumed three-dimensional Fermi surface. Finally, it is evident that the above difficulties cannot be solved by resorting to a strictly two-dimensional LDA, since the system becomes homogeneous and time independent when strictly projected in the $x-y$ plane.

In view of the above difficulties, it is clearly of great interest to test the validity of the 3D-LDA against a more direct calculation of the damping rate of a collective oscillation-a calculation that explicitly takes into account the reduced dimensionality of the quantum well. Our idea is to study a particularly simple oscillation that is described, to zero order in the Coulomb interaction, by a separable wave function, i.e., the product of an assigned time-dependent function of the $z$ coordinate of the electrons times a function of the $x$ and $y$ coordinates: the latter may be either the ground state or a uniform excited state of the quasi-2DEG in the $x-y$ plane. For example, we can put all the electrons in a given time-dependent combination of the lowest and the first excited subband: we assume that there is an external timedependent potential that can do this. The main physical assumption here is that all the electrons populate a single timedependent subband, and no intersubband scattering is considered. The time-dependent electronic density $n(z, t)$ creates, via time-dependent form factors, a time-dependent Coulomb field which induces transitions between different states of the 2DEG. We can make use of the Fermi golden rule to calculate the rate at which the motion in the $z$ direction creates electron-hole pairs in the $x-y$ plane. From this, we calculate the rate at which energy is transferred from the oscillatory motion in the $z$ direction to electron-hole pair excitations in the $x-y$ plane. In brief, the electron-hole pairs in the $x-y$ plane act as a thermal bath for the motion in the $z$ direction. Obviously, this approach is justified in the limit of weak Coulomb interaction (high density).

It turns out that it is possible to recast the results for the damping rate in a form that is analogous to the LDA, except that it involves a nonlocal viscosity $\zeta\left(z, z^{\prime}\right)$. This nonlocal (a)

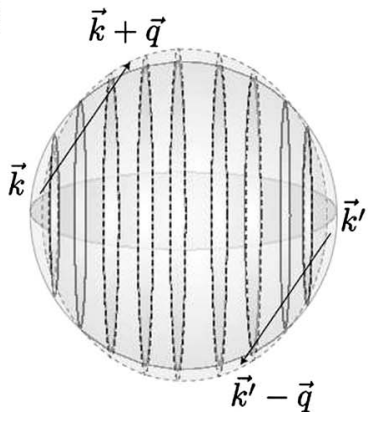

(b)

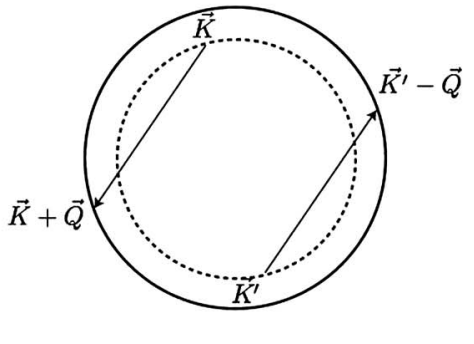

(c)

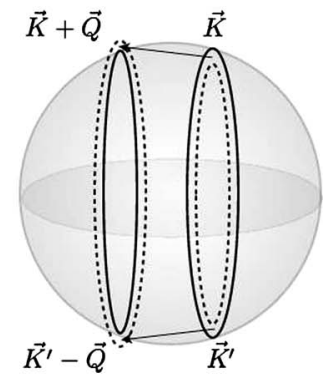

FIG. 2. (a) In a three-dimensional collective mode, the Fermi surface changes shape periodically from oblate to prolate. The process can be pictured as a transfer of electrons between the Fermi disks into which the Fermi surface can be sliced, perpendicular to the direction of propagation of the mode. These excitations are responsible for the finite shear viscosity of the electron liquid. (b) In two dimensions, for an isotropic single band system the Fermi surface is a circle: excitations can only change the radius of the circle, not its shape. There is no shear viscosity. (c) In multiband isotropic 2D systems, interband transitions allow a change in shape of the Fermi surface-formed in this case by several parallel disks: the shear viscosity reappears (2D wave vectors are represented by capital letters). 
viscosity can be converted into an effective local viscosity $\zeta(z)$ by integrating over $z^{\prime}$ at fixed $z$-a procedure that amounts to neglecting the finite range of nonlocality on the scale of variation of the density. In this manner, we are able to compare the approximate LDA viscosity $Y(z)$ - a function of the local equilibrium density-with the more accurate one, $\zeta(z)$.

We find significant differences between $\zeta(z, \omega)$ and $Y(z, \omega)$. At low frequency, the latter is dominated by a shear term, which is absent in $\zeta$. For this reason, $\zeta$ turns out to be numerically much smaller than $Y$. At high frequency $\zeta(z, \omega)$ and $Y(z, \omega)$ exhibit different power-law behaviors $\left(\omega^{-3}\right.$ and $\omega^{-5 / 2}$, respectively), reflecting different spectral densities of electron-hole excitations in two and three dimensions.

We evaluate the damping rate as a function of the frequency of the external field $\omega$ for the screened and unscreened potentials. We find, as one could expect, that the rate of energy dissipation in the screened (unscreened) interaction goes as $\omega^{4}\left(\omega^{2}\right)$ at small frequencies. In the 3D-LDA approximation, instead, the energy dissipation rate goes as $\omega^{2}$. Thus, the 3D-LDA largely overestimates the energy dissipation.

It is already known ${ }^{16}$ that the LDA viscosity is spurious in small systems such as atoms and molecules. The present findings show that a serious loss of accuracy can also occur in infinite systems, when the motion is restricted to a single one-dimensional subband as in the example considered here. Therefore, one needs to apply special care when using 3DLDA approximations in specific systems such as the one we consider here. The development of a better approximation for the viscous stress tensor-an approximation that is uniformly applicable across different dimensionalities-thus emerges as a critical issue.

\section{MODEL}

The Hamiltonian for an interacting $N$-electron system confined to a quantum well $(\mathrm{QW})$ of width $L$ in the $z$ direction is

$$
\hat{H}=\sum_{i} \frac{\hat{p}_{i}^{2}}{2 m}+V\left(\hat{r}_{i}\right)+\frac{1}{2} \sum_{i \neq j} v\left(\left|\hat{r}_{i}-\hat{r}_{j}\right|\right),
$$

where $v(r)=e^{2} / 4 \pi \epsilon|r|$ is the Coulomb potential ( $\epsilon$ is the background dielectric constant) and

$$
V(r) \equiv V(z)= \begin{cases}0, & |z|<L / 2 \\ \infty, & |z|>L / 2 .\end{cases}
$$

In the absence of electron-electron interactions $[v(r) \equiv 0]$, we can separate the Hamiltonian into two parts describing, respectively, the motion in the $z$ direction and the motion of the 2DEG in the plane:

$$
\hat{H}_{0}=\hat{H}_{z}+\hat{H}_{2 D E G},
$$

where

$$
\hat{H}_{z}=\sum_{i=1}^{N} \frac{p_{z, i}^{2}}{2 m}+V\left(\hat{z}_{i}\right)+V_{e x t}(\hat{z}, t)
$$

$$
\hat{H}_{2 D E G}=\sum_{i=1}^{N} \frac{p_{x, i}^{2}+p_{y, i}^{2}}{2 m} \equiv \sum_{i=1}^{N} \frac{P_{i}^{2}}{2 m},
$$

where $V_{e x t}(z, t)$ is the external potential that excites the QW (see the following discussion). Obviously, the Coulomb interaction couples these two subsystems in a nontrivial way. In the following, we will consider the Coulomb interaction as a small perturbation and then apply time-dependent perturbation theory to study the energy exchange between the $z$ motion and the density fluctuations of the 2DEG. In particular, we will show that, if one assumes a given motion in the $z$ direction, the Coulomb interaction excites electron-hole pairs in the $x-y$ plane, thus effectively transferring energy from the $z$ motion to the 2DEG.

A solution to the Schrödinger equation with the unperturbed Hamiltonian $\hat{H}_{0}$ can be written as the product of solutions of $H_{2 D E G}$ and $H_{z}$ in the following manner:

$$
|\Psi\rangle=|\Phi\rangle_{2 D E G}|\chi\rangle_{z},
$$

where $|\Phi\rangle_{2 D E G}$ is a Slater determinant of $N$ plane waves in the coordinates $R_{i}$ and

$$
|\chi\rangle_{z}=|\chi\rangle_{1}|\chi\rangle_{2} \cdots|\chi\rangle_{N}
$$

is a symmetric function of the $z$ coordinates of the electrons. We assume that all the electrons share a common motion in the $z$ direction specified by the one-electron wave function $\chi(z, t)$, which is sustained by a suitable time-dependent external potential $V_{e x t}(z, t)$. The density remains uniform in the $x-y$ plane. The antisymmetry of the complete wave function is ensured by the antisymmetry of $|\Phi\rangle_{2 D E G}$.

Next, we turn on the electron-electron interaction and derive an effective Hamiltonian for the dynamics of $|\Phi\rangle_{2 D E G}$ in the presence of the driving potential $V_{e x t}(z, t)$. This is done by substituting Eqs. (8) and (9) into the Schödinger equation

$$
i \hbar \partial_{t}|\Psi(t)\rangle=\hat{H}(t)|\Psi(t)\rangle .
$$

Making use of the Fourier representation of the Coulomb potential,

$$
v(\hat{r})=\frac{e^{2}}{\epsilon V} \sum_{q, Q} \frac{e^{-i q \hat{z}} e^{-i Q \cdot \hat{R}}}{q^{2}+Q^{2}}
$$

$[R$ and $Q$ are vectors in the $2 \mathrm{D}$ real space and momentum space respectively, with $r=(R, z), q$ is a one-dimensional momentum, and $V$ the volume], and the Fourier transform of the density

$$
n(q, t)=\int_{-L / 2}^{L / 2} d z e^{i q z} n(z, t),
$$

we can write the electron-electron interaction as

$$
\hat{H}_{e e}(t)=\frac{e^{2}}{2 \epsilon V} \sum_{i \neq j} \sum_{q, Q} \frac{e^{-i q\left(\hat{z}_{i}-\hat{z}_{j}\right)}}{q^{2}+Q^{2}} e^{-i Q \cdot\left(\hat{R}_{i}-\hat{R}_{j}\right)} .
$$

Assuming that the total wave function is still approximately of the form (8), we can ignore the correlation between the $z$ 's and the $R$ 's, and simply replace $e^{-i q\left(\hat{z}_{i}-\hat{z}_{j}\right)}$ by its average value in the given one-electron wave function (9): 


$$
e^{-i q\left(\hat{z}_{i}-\hat{z}_{j}\right)} \rightarrow\left\langle e^{-i q\left(\hat{z}_{i}-\hat{z}_{j}\right)}\right\rangle=|n(q, t)|^{2},
$$

independent of $i$ and $j$. Then, the effective dynamics for $|\Phi\rangle_{2 D E G}$ is given by

$$
i \hbar \partial_{t}|\Phi\rangle_{2 D E G}=\hat{H}_{e f f}|\Phi\rangle_{2 D E G},
$$

where

$$
\hat{H}_{e f f}=\sum_{i=1}^{N} \frac{\hat{P}_{i}^{2}}{2 m}+\frac{e^{2}}{2 \epsilon V} \sum_{i \neq j} \sum_{q, Q} \frac{|n(q, t)|^{2}}{q^{2}+Q^{2}} e^{-i Q \cdot\left(\hat{R}_{i}-\hat{R}_{j}\right)} .
$$

Notice that $|n(q, t)|^{2}$ plays the role of a time-dependent form factor for the electron-electron interaction in the plane.

To apply perturbation theory, we assume that $n(q, t)$ is the sum of a static part plus a small dynamical contribution:

$$
n(q, t)=n_{0}(q)+\delta n(q, t),
$$

with $|\delta n(q, t)| \ll n_{0}(q)$ for all $q$ and $t$. Neglecting contributions of order $(\delta n)^{2}$, we get the effective time-dependent Schrödinger equation for $|\Phi\rangle_{2 D E G}$,

$$
\begin{aligned}
& {\left[i \hbar \partial_{t}-\sum_{i=1}^{N} \frac{\hat{P}_{i}^{2}}{2 m}-\frac{e^{2}}{2 \epsilon V} \sum_{i \neq j} \sum_{q, Q} \frac{\left|n_{0}(q)\right|^{2}}{q^{2}+Q^{2}} e^{-i Q \cdot\left(\hat{R}_{i}-\hat{R}_{j}\right)}\right.} \\
& \left.\quad-\frac{e^{2}}{\epsilon V} \sum_{i \neq j} \sum_{q, Q} \frac{\operatorname{Re}\left[n_{0}(q) \delta n(-q, t)\right]}{q^{2}+Q^{2}} e^{-i Q \cdot\left(\hat{R}_{i}-\hat{R}_{j}\right)}\right]|\Phi\rangle_{2 D E G}=0 .
\end{aligned}
$$

The left-hand side of Eq. (18) is the sum of a static part and a time-dependent part. The static part includes the effect of the QW static form factor on the ground state and the excited states of the 2DEG. We assume that the 2DEG is in its ground state at $t=0$. The time-dependent part induces transitions between eigenstates of the static 2DEG Hamiltonian and thus is responsible for dissipation.

An important check for this model is that if the motion in the quantum well is a rigid translation of the equilibrium density in the $z$ direction (Kohn mode), i.e., $n(z, t)=n_{0}(z$ $+u(t)) \simeq n_{0}(z)+\partial_{z} n_{0}(z) u(t)$, where $u(t)$ is a spatially uniform function of time, then the dissipation must be absent. This is easily verified from Eq. (18): for the Kohn mode, we have $\delta n(q, t)=i q n_{0}(q) u(t)$, where $u(t)$ is a real function, and

$$
\operatorname{Re}\left[n_{0}(q) \delta n(-q, t)\right]=\operatorname{Re}\left[i q\left|n_{0}(q)\right|^{2} u(t)\right]=0 .
$$

Therefore, only the static nondissipative part of the Hamiltonian survives for the Kohn mode.

\section{PERTURBATION THEORY}

To apply the Fermi golden rule, we assume that the forcing density $\delta n(z, t)$ is a periodic function of time with angular frequency $\omega, \delta n(q, t)=\delta n(q, \omega) e^{-i \omega t}+$ c.c., and we write

$$
\delta n(q, \omega)=\frac{q j(q, \omega)}{\omega},
$$

where $j(q, \omega)$ is the Fourier amplitude of the current,

$$
n_{0}(z) \partial_{t} u(z, t)=j(q, \omega) e^{i(q z-\omega t)}+\text { c.c. }
$$

The energy transferred per unit of time by the $z$ motion to the 2 DEG is equal to the energy $\hbar \omega$ absorbed in each allowed transition from the ground state to an excited state of the 2DEG times the rate at which the transition occurs. Evidently, the only allowed transitions in leading order are the ones in which two electron-hole pairs with opposite wave vectors $Q$ and $-Q$ are created in the 2DEG, with energies adding up to $\hbar \omega$. The first pair is created by promoting an electron from state $K$ within the two-dimensional Fermi surface of the 2DEG to state $K+Q$ outside the Fermi surface. Similarly, the second pair is created by promoting an electron from $K^{\prime}$ to $K^{\prime}-Q$, with $K^{\prime}$ inside and $K^{\prime}-Q$ outside the Fermi surface [see Fig. 2, panel (b)]. So the energy absorbed per unit time is

$$
\begin{aligned}
\frac{d E}{d t}= & \frac{4 \pi e^{4}}{\epsilon^{2} \hbar \mathcal{A}} \sum_{K, K^{\prime}<K_{F}} \int d^{2} Q \int d q \int d q^{\prime} \delta\left(\omega-\omega_{K, K^{\prime}, Q}\right) \\
& \times \frac{q q^{\prime}}{\omega} n_{0}(q) j(-q, \omega) \frac{\left(1-n_{K+Q}\right) n_{K}\left(1-n_{K^{\prime}-Q}\right) n_{K^{\prime}}}{q^{2}+Q^{2}} \\
& \times\left[\frac{1}{q^{\prime 2}+Q^{2}}-\frac{1}{q^{\prime 2}+\left(Q+K-K^{\prime}\right)^{2}}\right] n_{0}^{*}\left(q^{\prime}\right) j^{*}\left(-q^{\prime}, \omega\right),
\end{aligned}
$$

where $n_{K}$ is the Fermi occupation number $\left[n_{K}=\Theta\left(K_{F}-K\right)\right.$, where $\Theta(x)$ is the step function and $K_{F}$ is the Fermi wave vector $]$ and $\omega_{K, K^{\prime}, Q} \equiv \hbar\left[Q^{2}+Q \cdot\left(K^{\prime}-K\right)\right] / m$ is the energy of the double electron-hole pair. $\mathcal{A}$ is the area occupied by the 2DEG. The two terms in the square bracket arise from direct and exchange processes, respectively - the latter arising from the antisymmetry of the 2DEG wave function. In the following, we will disregard the exchange term. Aside from the fact that its contribution is small in the high-density limit, we must keep in mind that the exchange contribution was also dropped in previous calculations of the LDA viscosity: ${ }^{27}$ for a fair comparison, we must drop it here too.

Introducing in Eq. (22) the imaginary part of the twodimensional Lindhard function, ${ }^{3}$

$$
\Im m \chi_{0}^{2 D}(Q, \omega)=-\frac{\pi}{\hbar \mathcal{A}} \sum_{K} n_{K}\left(1-n_{K+Q}\right) \delta\left(\omega-\omega_{K, Q}\right),
$$

where $\omega_{K, Q}=\hbar\left[Q^{2} / 2 m-Q \cdot K / m\right]$, we obtain

$$
\begin{aligned}
\frac{d E}{d t}= & \frac{e^{4} \hbar \mathcal{A}}{4 \pi^{4} \epsilon^{2} \omega} \int d q q j(-q, \omega) n_{0}(q) \\
& \times \int d q^{\prime} q^{\prime} j^{*}\left(-q^{\prime}, \omega\right) n_{0}^{*}\left(q^{\prime}\right) \int d^{2} Q \int_{0}^{\omega} d \omega^{\prime} \\
& \times \frac{\left.\Im m \chi_{0}^{2 D}\left(Q, \omega^{\prime}\right) \Im m \chi_{0}^{2 D}\left(Q, \omega-\omega^{\prime}\right)\right]}{\left(q^{2}+Q^{2}\right)\left(q^{\prime 2}+Q^{2}\right)} .
\end{aligned}
$$

Notice that $\chi_{0}^{2 D}$ depends only on the sheet density of the electron gas, which is independent of $z$. 
As a last step, we express this result in terms of the velocity field, $v(z, t)=\partial_{t} u(z, t)=j(z, t) / n_{0}(z)$,

$$
\frac{d E}{d t}=-2 \mathcal{A} \int d z \int d z^{\prime} \partial_{z} v(z, \omega) \zeta\left(z, z^{\prime} ; \omega\right) \partial_{z^{\prime}} v^{*}\left(z^{\prime}, \omega\right),
$$

where

$$
\begin{aligned}
\zeta\left(z, z^{\prime} ; \omega\right)= & n_{0}(z) n_{0}\left(z^{\prime}\right) \frac{\hbar}{4 \pi \omega} \int_{0}^{\omega} d \omega^{\prime} \int d Q Q \widetilde{v}(Q, z) \\
& \times \widetilde{v}^{*}\left(Q, z^{\prime}\right) \Im m \chi_{0}^{2 D}\left(Q, \omega^{\prime}\right) \Im m \chi_{0}^{2 D}\left(Q, \omega-\omega^{\prime}\right)
\end{aligned}
$$

is the nonlocal bulk viscosity of the electron gas in the QW and

$$
\widetilde{v}(Q, z)=\frac{2 e^{2}}{\epsilon} \int_{-\infty}^{\infty} d q \frac{n_{0}(q) e^{-i q z}}{q^{2}+Q^{2}}
$$

the effective electron-electron interaction in the plane of the 2DEG.

Equation (25) should be compared with the corresponding LDA expression,

$$
\begin{aligned}
\left.\frac{d E}{d t}\right|_{L D A}= & -2 \mathcal{A} \int d z\left|\partial_{z} v(z, \omega)\right|^{2} \\
& \times\left[\zeta^{L D A}(z ; \omega)+\frac{4}{3} \eta^{L D A}(z, \omega)\right],
\end{aligned}
$$

where (see Refs. 27 and 28)

$$
\begin{aligned}
\zeta^{L D A}(z, \omega)= & \frac{\hbar}{36 \pi^{2} \omega} \int_{0}^{\omega} d \omega^{\prime} \int d q q^{2}|v(q)|^{2} \\
& \times \Im m \chi_{0}^{3 D}\left(q, \omega^{\prime}\right) \Im m \chi_{0}^{3 D}\left(q, \omega-\omega^{\prime}\right), \\
\eta^{L D A}(z, \omega)= & \frac{4 \hbar}{15 \pi^{2} \omega} \int_{0}^{\omega} d \omega^{\prime} \int d q|v(q)|^{2} q^{2} \Im m \chi_{0}^{3 D}\left(q, \omega^{\prime}\right) \\
& \times \Im m \chi_{0}^{3 D}\left(q, \omega-\omega^{\prime}\right)+\frac{\hbar}{5 \pi^{2} \omega^{3}} \\
& \times \int_{0}^{\omega} d \omega^{\prime} \int d q|v(q)|^{2} q^{4} \Im m \chi_{0 T}^{3 D}\left(q, \omega^{\prime}\right) \\
& \times \Im m \chi_{0}^{3 D}\left(q, \omega-\omega^{\prime}\right),
\end{aligned}
$$

$\chi_{0}^{3 D}(q, \omega)$ is the 3D Lindhard function, $\chi_{0 T}^{3 D}(q, \omega)$ is the noninteracting transverse current-current response function in $3 \mathrm{D}$, and $v(q)$ is the Fourier transform of the screened 3D Coulomb interaction (about the screening more will be said later). The $z$ dependence of these functions arises from the fact that the 3D Lindhard functions depend on the local density.

To facilitate the comparison between the two sets of formulas (25), (26), and (28)-(30), we introduce the integrated viscosity

$$
\zeta(z, \omega) \equiv \int \zeta\left(z, z^{\prime} ; \omega\right) d z^{\prime},
$$

which has the same physical dimensions as $\zeta^{L D A}(z, \omega)$ (energy $\times$ time/volume) and can be directly compared to it. The explicit expression for $\zeta(z, \omega)$ is

$$
\begin{aligned}
\zeta(z, \omega)= & n_{0}(z) \frac{\hbar \mathcal{A}}{4 \pi \omega} \int_{0}^{\omega} d \omega^{\prime} \int d Q Q \widetilde{v}(Q, z) \\
& \times \widetilde{v}^{*}(Q) \Im m \chi_{0}^{2 D}\left(Q, \omega^{\prime}\right) \Im m \chi_{0}^{2 D}\left(Q, \omega-\omega^{\prime}\right),
\end{aligned}
$$

where

$$
\widetilde{v}(Q) \equiv \int n_{0}(z) \widetilde{v}(Q, z) d z
$$

It is interesting to compare Eq. (32) with the expressions obtained from the xc kernel in the 3D-LDA [see Eq. (29)]. In the homogeneous 3D electron liquid, there are two independent viscosities: the bulk viscosity $\zeta(\omega)$ and the shear viscosity $\eta(\omega)$. The former arises from motions that change the volume of the local Fermi surface (the density), but not its shape. The latter appears when the motion changes the shape of the Fermi surface, even if its volume does not change. A collisionless longitudinal collective mode, such as the ordinary plasmon, involves both types of motion simultaneously, as discussed in the caption of Fig. 2: the effective viscosity for such a mode is $Y(\omega) \equiv \zeta^{L D A}(\omega)+\frac{4}{3} \eta^{L D A}(\omega){ }^{25,26} Y$ can be easily constructed from the combination of the two equations, Eqs. (29) and (30).

Aside from the obvious difference in the dimensionality of the Lindhard functions, we see that our expression (32) for $\zeta(z, \omega)$ is formally similar to expression (29) for $\zeta^{L D A}(\omega)$ in 3D-LDA. These expressions, however, vanish as $\omega^{2}$ at low frequency, because the Lindhard spectra $\Im m \chi_{0}^{2 D(3 D)}(q, \omega)$ vanish as $\omega$. As a result $\zeta(z, \omega)$, as well as $\zeta^{L D A}(\omega)$, goes as $\omega^{2}$ at low frequency. On the other hand, the behavior of the LDA shear viscosity $\eta^{L D A}(\omega)$ is quite different. From Eq. (30), we see that this quantity contains a term involving the transverse current spectrum $\Im m \chi_{0 T}^{3 D}$, and this term tends to a finite limit for $\omega \rightarrow 0$ because the factor $1 / \omega^{3}$ compensates for the smallness of the Lindhard spectra.

Therefore, a fundamental difference exists between our results and those of the 3D-LDA in the low-frequency regime. While the LDA viscosity is dominated by the shear term, which remains finite for $\omega \rightarrow 0$, the present viscosity is purely of the bulk type and vanishes as $\omega^{2}$ for $\omega \rightarrow 0$, at least when the screening of the electron-electron interaction is properly taken into account (see discussion below). Physically, the absence of a shear term is due to the fact that the oscillatory motion in the $z$ direction does not change the shape of the local Fermi surface of the 2DEG (a circle), whereas in a 3D oscillation the local Fermi surface changes its shape periodically from a prolate to an oblate ellipsoid (passing through the sphere), generating shear friction in the process (see Fig. 2).

Before proceeding to a detailed comparison of the numerical results for the viscosities and the energy dissipation rates, we need to say something more about the role of the 

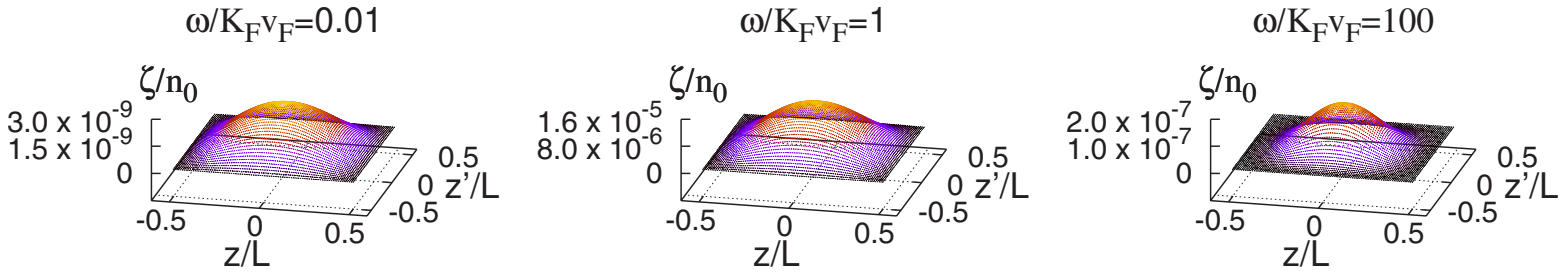

FIG. 3. (Color online) The nonlocal viscosity $\zeta\left(z, z^{\prime}, \omega\right) / n_{0}$ as a function of $z, z^{\prime}$ for different values of $\omega$ with $K_{F} L=3.17$ and screened interaction. The nonlocal viscosity for the unscreened interaction has similar shape but could differ by a few orders of magnitude in amplitude (see also Fig. 6) especially at small frequencies due to the singularity of the Coulomb interaction at small momenta.

screening of the electron-electron interaction. First of all, it must be noticed that the integrals over wave vector in Eqs. (29) and (30) diverge, in the limit $\omega \rightarrow 0$, if $v(q)$ is taken to be the Fourier transform of the bare Coulomb interaction, $4 \pi e^{2} / \epsilon q^{2}$. The divergence comes from the small $q$ region. The standard cure for this type of divergence is to screen the interaction by the static dielectric function of the electron gas, which, in the high-density limit, can be reasonably approximated by the Thomas-Fermi formula. The screened interaction has the form

$$
v(q)=\frac{4 \pi e^{2}}{\epsilon\left(q^{2}+\kappa^{2}\right)},
$$

where $\kappa=\sqrt{4 \pi e^{2} N(0)}$ is the Thomas-Fermi wave vector and $N(0)$ is the density of states at the Fermi energy.

The situation is somewhat different in Eq. (32). Because the effective interaction $\widetilde{v}(Q, z)$ diverges at small $Q$ only as $\frac{2 \pi e^{2}}{Q}$, it turns out that the wave-vector integral is finite even without including the screening. Nevertheless, the integral has a large contribution from extremely small wave vectors, of order $\omega / v_{F}$ for $\omega \rightarrow 0$, which effectively changes the formally expected $\omega^{2}$ behavior of $\zeta(z, \omega)$ to a constant independent of $\omega$. This curious phenomenon is shown in Fig. 4, where we see that $\zeta(z, \omega)$ tends to a constant when the interaction is not screened. Inclusion of screening, however, will drastically modify this behavior, reinstating the expected $\omega^{2}$ behavior (see Fig. 5). To demonstrate this point, we have calculated $\zeta(z, \omega)$ with the bare interaction $\widetilde{v}(Q, z)$ replaced by a screened interaction according to the scheme

$$
\widetilde{v}(Q, z) \rightarrow \frac{\widetilde{v}(Q, z)}{1-\widetilde{v}(Q) \chi_{0}(Q, 0)}
$$

The denominator is the static dielectric constant of a $2 \mathrm{D}$ QW in which all the electrons reside in the lowest subband, and no intersubband transitions are allowed. Indeed, the lowenergy excitations of the 2DEG are exclusively intrasubband electron-hole pairs. Therefore, neglecting intersubband transitions is justified at low frequency and qualitatively correct at higher frequencies (as long as only a few subbands are involved).

\section{NUMERICAL RESULTS}

Figure 3 shows the calculated values of the nonlocal viscosity $\zeta\left(z, z^{\prime}, \omega\right)$ as a function of $z$ and $z^{\prime}$ for a sheet density $n_{2 D}=10^{11} \mathrm{~cm}^{-2}$ and $L=40 \mathrm{~nm} \quad\left(K_{F}=\sqrt{2 \pi n_{2 D}}=7.9\right.$ $\times 10^{5} \mathrm{~cm}^{-1}$ ) for the case of screened interaction. We have assumed that the equilibrium density in the QW has the form

$$
n_{0}(z)=\frac{2}{L} \cos ^{2}\left(\frac{\pi z}{L}\right)
$$

appropriate to the lowest subband of a free particle in a box. This choice gives

$$
\widetilde{v}(Q, z)=-\frac{2 \pi e^{2} L}{\epsilon(L Q)^{2}}\left[\frac{e^{-Q L / 2} \cosh (Q z)}{1+\frac{(L Q)^{2}}{4 \pi^{2}}}-\frac{\cos \left(\frac{2 \pi z}{L}\right)}{1+\frac{4 \pi^{2}}{(Q L)^{2}}}-1\right]
$$

and, for the unscreened interaction,

$$
\begin{aligned}
\widetilde{v}(Q)= & -\frac{2 \pi e^{2}}{\epsilon Q}\left[\frac{2 e^{-Q L / 2} \sinh (Q L / 2)}{(Q L)^{2}\left(1+\frac{(Q L)^{2}}{4 \pi^{2}}\right)^{2}}\right. \\
& \left.-\frac{1}{Q L}-\frac{Q L}{16 \pi^{2}\left(1+\frac{(Q L)^{2}}{2 \pi^{2}}\right)}\right] .
\end{aligned}
$$

The functions $\widetilde{v}(Q, z)$ and $\widetilde{v}(Q)$ depend on the physical parameter $K_{F} L$, where $K_{F}$ is the Fermi momentum of the 2DEG and $L$ is the thickness of the well in the $z$ direction. For $K_{F} L \ll 1, \widetilde{v}(Q, z) \simeq \widetilde{v}(Q) \simeq 2 \pi e^{2} / \epsilon Q$, i.e., the functions $\widetilde{v}$ reduce to Fourier transforms of the bare two-dimensional Coulomb potential.

The nonlocal viscosity $\zeta\left(z, z^{\prime}, \omega\right) /\left[n_{0}(0) \hbar\right]$ decays when one of the points is close to the boundary and it is maximum when both $z$ and $z^{\prime}$ are near the center of the well. While the spatial behavior of $\zeta\left(z, z^{\prime}, \omega\right)$ looks qualitatively similar for the screened and unscreened cases, the order of magnitude is quite different especially at small frequencies, the unscreened results being quite large. The shape of the nonlocal viscosity is dominated by the ground-state densities $n_{0}(z)$ and $n_{0}\left(z^{\prime}\right)$ [see Eq. (26)]: The function $\zeta\left(z, z^{\prime}, \omega\right) / n_{0}(z) n_{0}\left(z^{\prime}\right)$ is a slowly varying function of the positions $z$ and $z^{\prime}$. However, the magnitude of this function depends strongly on whether we use a screened or an unscreened interaction. This 


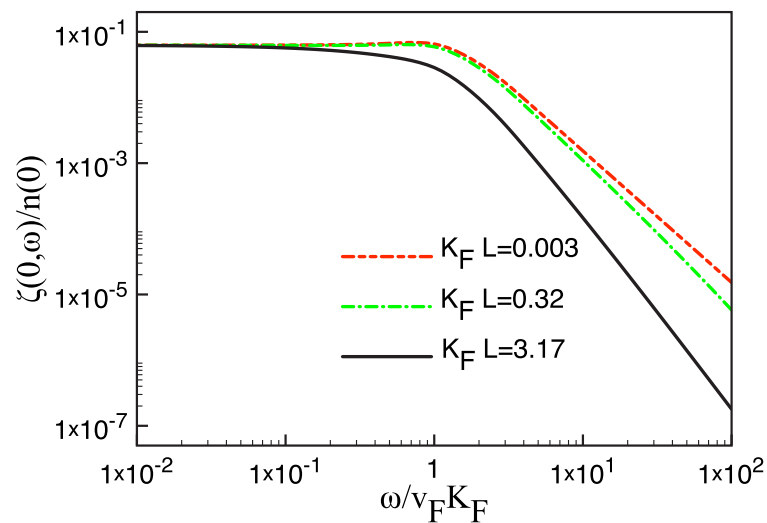

FIG. 4. (Color online) Average local viscosity $\zeta(z, \omega) / \hbar n_{0}(z)$ from Eq. (32) as a function of frequency for $z=0$ and for different values of $K_{F} L$. The Coulomb interaction potential is unscreened. Notice that $\zeta$ approaches a finite value for $\omega \rightarrow 0$.

has its origin in the strong singularity of the Coulomb potential for small momenta. We then expect that in the case of the unscreened interaction, the dissipation rate is largely overestimated, especially at small frequencies when the screening is more effective.

In Fig. 4, we plot the integrated viscosity $\zeta(0, \omega)$ for various values of $K_{F} L$, as a function of the frequency for the unscreened interaction. For all the values of the parameter $K_{F} L$, we notice the existence of a finite limiting value for small frequencies. As already discussed, this finite limiting value is due to the existence of a strong singularity for small $Q, 1 / Q^{3}$, which compensates for the smallness of the Lindhard spectra. Including the screening according to the scheme of Eq. (34) changes this behavior dramatically. The new behavior is shown in Fig. 5 for various values of the parameter $K_{F} L$. Now, $\zeta(0, \omega)$ vanishes as $\omega^{2}$, in agreement with the general discussion of the previous section.

Finally, in Fig. 6 we compare the behavior of $\zeta(0, \omega)$ with the corresponding LDA quantity $Y(\omega)$, evaluated at the density $n_{0}(z=0)$. In the screened case, our viscosity falls well below $Y(\omega)$ (calculated from the parametrization of Qian and Vignale $\left.{ }^{28}\right)$, demonstrating the intrinsic limitation of the 3DLDA in this regime.

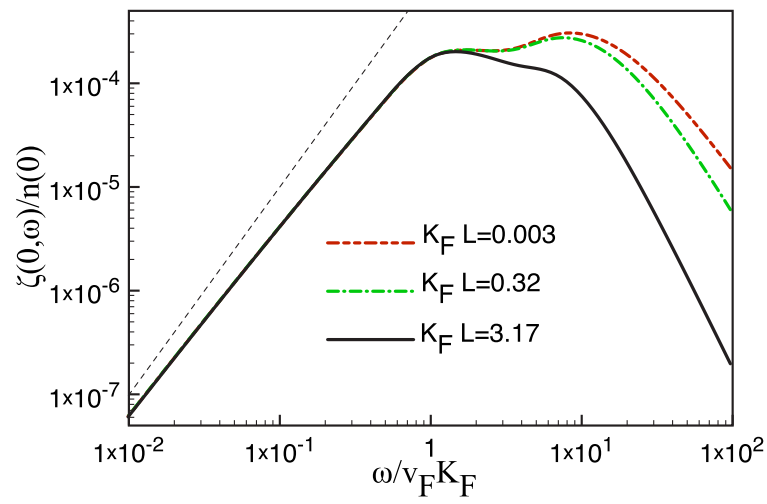

FIG. 5. (Color online) Same as Fig. 4, but now for the case of a screened Coulomb interaction. Notice that $\zeta(0, \omega) \propto \omega^{2}$ for $\omega \rightarrow 0$ (the dashed line is the slope of $\omega^{2}$ in the logarithmic plot).

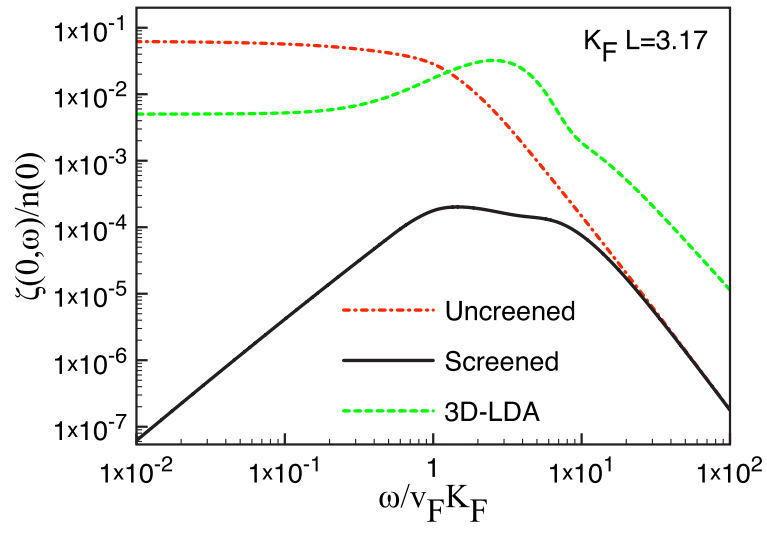

FIG. 6. (Color online) Comparison between $\zeta(z, \omega) / \hbar n_{0}(z)$ and the 3D-LDA viscosity $Y(z, \omega)$ for $z=0$. As expected, the screened and unscreneed results for $\zeta(z, \omega)$ coincide for large frequencies. Notice that the 3D-LDA (with a screened interaction) predicts a finite limit of the viscosity for $\omega \rightarrow 0$.

It is also interesting-although not fully justified physically-to consider the high-frequency behavior of $\zeta(z, \omega)$ vis-a-vis the high-frequency behavior of $Y(\omega)$. This can be calculated analytically and one sees that the relevant contribution to the integral over wave vector comes from $Q$ proportional to $\sqrt{2 m \omega} / \hbar$ and is therefore large. In this regime, both $\widetilde{v}(Q)$ and $v(q)$ scale as $\omega^{-1}$, so the only difference between expressions (32) and (29) comes from the different "volume element" in wave-vector space, $Q$ and $q^{2}$, respectively. Taking this into account, we immediately understand the origin of the $\sqrt{\omega}$ difference in the high-frequency behaviors of $\zeta(z, \omega)\left(\omega^{-3}\right)$ and $Y(\omega)\left(\omega^{-5 / 2}\right)$ - the former tending to zero faster than the latter.

To fully evaluate the energy dissipation via either Eq. (25) or Eq. (28), we need to assign the velocity $v(z, \omega)$. In our model, we consider coherent oscillations of the wave function in the quantum well. For this motion, we then choose

$$
\chi(z, t)=\chi_{1}(z)+\lambda e^{i \omega t} \chi_{3}(z)
$$

where $\lambda$ is a constant and $\chi_{n}(z)$ are the normalized eigenfunctions of the quantum well,

$$
\chi_{n}(z)=\sqrt{\frac{2}{L}} \begin{cases}\sin \left(\frac{n \pi z}{L}\right), & n \text { even } \\ \cos \left(\frac{n \pi z}{L}\right), & n \text { odd }\end{cases}
$$

For the linear-response theory to be valid, we have assumed $\lambda \ll 1{ }^{29}$ We can evaluate the current density for the state $\chi(z, t)$ and obtain the velocity field

$$
\partial_{z} v(z, \omega)=\partial_{z} \frac{j(z, \omega)}{n_{0}(z)}=-\omega|\lambda| \cos \left(\frac{2 \pi z}{L}\right) .
$$

By substituting this expression in the equation for the energy dissipation [Eq. (25)], we get 


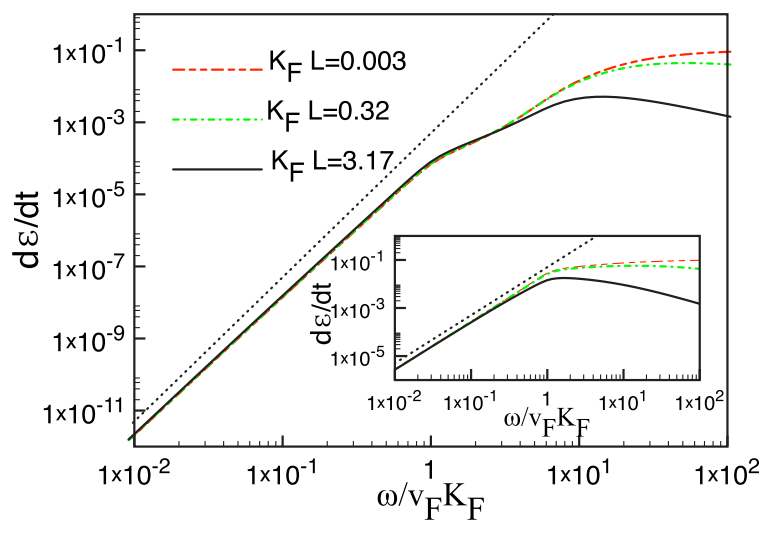

FIG. 7. (Color online) The energy dissipation rate for the screened and unscreened (inset) interactions [see Eq. (40)] as a function of the external frequency for various values of the quantity $K_{F} L$. The energy dissipation rate scales as $\omega^{4}$ for the screened interaction, a result much smaller than the $\omega^{2}$ behavior obtained from the 3D-LDA energy dissipation rate (also for a screened interaction). The limiting behavior $d \mathcal{E} / d t \simeq \omega^{4}$ is shown for reference as a dotted line. Inset: Due to the strong singularity of the unscreened interaction, the energy dissipation rate for this case scales as $\omega^{2}$. The dotted line shows the limiting behavior $d \mathcal{E} / d t \simeq \omega^{2}$.

$$
\begin{aligned}
\frac{d E}{d t}= & -\frac{A \hbar}{\pi \omega} \int_{0}^{\omega} d \omega^{\prime} \int d Q Q \Im m \chi_{0}^{2 D}\left(Q, \omega^{\prime}\right) \\
& \times \Im m \chi_{0}^{2 D}\left(Q, \omega-\omega^{\prime}\right) \Gamma^{2}\left(Q, \omega^{\prime}\right),
\end{aligned}
$$

where we have defined for the unscreened case

$$
\begin{aligned}
\Gamma(Q, \omega)= & -\frac{e^{2} \omega}{2 \pi \epsilon} \frac{|\lambda| L}{1+\frac{(L Q)^{2}}{4 \pi^{2}}}\left[1+\frac{2 \pi^{2}}{(L Q)^{2}}\right. \\
& \left.+\frac{e^{Q L / 2} \sinh \left(\frac{Q L}{2}\right)}{2 Q L} \frac{1-\frac{8 \pi^{2}}{(L Q)^{2}}}{\left(1+\frac{(L Q)^{2}}{16 \pi^{2}}\right)\left(1+\frac{(L Q)^{2}}{4 \pi^{2}}\right)}\right] .
\end{aligned}
$$

According to the scheme we adopted [see Eq. (34)], to include screening in the interaction potential we substitute $\Gamma(Q, \omega)$ with $\Gamma(Q, \omega) /\left[1-\chi_{0}(Q) \widetilde{v}(Q)\right]$.

In Fig. 7, we plot $d \mathcal{E} / d t=d / d t\left(E / N \hbar \omega_{p}^{2}|\lambda|^{2}\right)$, where $\omega_{p}^{2}$ $=2 \pi n_{0}(0) e^{2} K_{F} / m$ is the 2D plasmon frequency evaluated at the Fermi momentum, by directly evaluating Eq. (40) for the screened and unscreened cases. It is immediately seen that for small frequencies, the energy dissipation rate scales as $\omega^{4}$ $\left(\omega^{2}\right)$ for the screened (unscreened) interaction. This has to be compared with the energy dissipation rate obtained from the 3D-LDA: since $Y(\omega)$ goes to a constant for small frequencies (see Fig. 6), we see that the 3D-LDA dissipation rate scales as $\omega^{2}$ for small $\omega$. Then, we can conclude that the 3D-LDA overestimates the energy dissipation rate in our model.

\section{DISCUSSION}

The system studied in this paper can be thought of as consisting of two weakly coupled subsystems of reduced dimensionality: a linear oscillator in the $z$ direction coupled to a two-dimensional electron gas in the $x-y$ plane. We have calculated the energy transfer between these two subsystems to leading order in the strength of their coupling and we have thus identified a nonlocal viscosity which can be compared (after the integration of one variable) with the viscosity obtained from the standard 3D-LDA. We have found very significant differences between the two viscosities, particularly at low frequency, where the 3D-LDA viscosity is dominated by a shear term which is absent in the present treatment. This point is clearly shown in Fig. 6, where we compare the effective viscosity as obtained within LDA and the bulk viscosity we obtain from perturbation theory. We conclude that LDA grossly overestimates the viscosity in this system. This error is also evident in Fig. 7, where we plot the energy dissipation calculated in our model with the exact expression for $\zeta$. The 3D-LDA energy rate dissipation goes as $\omega^{2}$ for small $\omega$, while the result from perturbation theory scales as $\omega^{4}$, reflecting the reduced bulk viscosity in that regime.

It is important to realize that the problem we have uncovered stems from the extreme one-dimensional character of the dynamics of our model. We have assumed that all the electrons reside in a single time-dependent subband $\chi(z, t)$. In effect, this dramatically limits the type of excitations we can generate in the 2DEG (see Fig. 2) and this is the reason why we do not have the transverse current term that is responsible, in three dimensions, for the shear viscosity term. It is expected that a transversal shear viscosity term will reappear in a multisubband system via the relative motion of the different sheets of the Fermi surface in different subbands. For a strictly one-dimensional motion, however, it seems clear that the use of the LDA is problematic. This leaves us with the challenge of formulating a better approximation to keep track of the systematic reduction in viscosity following from the geometric confinement of the system.

\section{ACKNOWLEDGMENTS}

R.D. thanks C. Ullrich for his assistance with part of the numerical calculations. R.D. and M.D.V. acknowledge financial support from the Department of Energy Grant No. DE-FG02-05ER46204. G.V. acknowledges financial support from the Department of Energy Grant No. DE-FG0205ER46203. 
*Electronic address: dagosta@physics.ucsd.edu

${ }^{1}$ E. Runge and E. K. U. Gross, Phys. Rev. Lett. 52, 997 (1984).

${ }^{2}$ Time-Dependent Density Functional Theory, Lecture Notes in Physics Vol. 706, edited by M. Marques, C. Ullrich, F. Nogueira, A. Rubio, K. Burke, and E. K. U. Gross (Springer, Berlin, 2006).

${ }^{3}$ S. K. Ghosh and A. K. Dhara, Phys. Rev. A 38, 1149 (1988).

${ }^{4}$ G. F. Giuliani and G. Vignale, Quantum Theory of the Electron Liquid (Cambridge University Press, Cambridge, 2005).

${ }^{5}$ P. Hohenberg and W. Kohn, Phys. Rev. 136, B864 (1964).

${ }^{6}$ W. Kohn and L. J. Sham, Phys. Rev. 140, A1133 (1965).

${ }^{7}$ R. van Leeuwen, Phys. Rev. Lett. 82, 3863 (1999).

${ }^{8}$ G. Vignale and W. Kohn, in Electronic Density Functional Theory: Recent Progress and New Directions, edited by J. F. Dobson, G. Vignale, and M. P. Das (Plenum, New York, 1996), p. 199.

${ }^{9}$ G. Vignale and W. Kohn, Phys. Rev. Lett. 77, 2037 (1996).

${ }^{10}$ G. Vignale, C. A. Ullrich, and S. Conti, Phys. Rev. Lett. 79, 4878 (1997).

${ }^{11}$ M. Di Ventra and R. D’Agosta, Phys. Rev. Lett. 98, 226403 (2007).

${ }^{12}$ R. D'Agosta and M. Di Ventra, J. Phys.: Condens. Matter 18, 11059 (2006).

${ }^{13}$ J. B. Williams, M. S. Sherwin, K. D. Maranowski, and A. C. Gossard, Phys. Rev. Lett. 87, 037401 (2001).

${ }^{14}$ C. A. Ullrich and G. Vignale, Phys. Rev. Lett. 87, 037402 (2001).

${ }^{15}$ C. A. Ullrich and G. Vignale, Phys. Rev. B 65, 245102 (2002).
${ }^{16}$ C. A. Ullrich and K. Burke, J. Chem. Phys. 121, 28 (2004).

${ }^{17}$ M. van Faassen, P. L. de Boeij, R. van Leeuwen, J. A. Berger, and J. G. Snijders, Phys. Rev. Lett. 88, 186401 (2002).

${ }^{18}$ J. A. Berger, P. L. de Boeij, and R. van Leeuwen, Phys. Rev. B 71, 155104 (2005).

${ }^{19}$ J. A. Berger, P. L. de Boeij, and R. van Leeuwen, Phys. Rev. B 75, 035116 (2007).

${ }^{20}$ N. Sai, M. Zwolak, G. Vignale, and M. Di Ventra, Phys. Rev. Lett. 94, 186810 (2005).

${ }^{21}$ R. D’Agosta, N. Sai, and M. Di Ventra, Nano Lett. 6, 2935 (2006).

${ }^{22}$ N. Sai, N. Bushong, R. Hatcher, and M. Di Ventra, Phys. Rev. B 75, 115410 (2007).

${ }^{23}$ P. Romaniello and P. L. de Boeij, Phys. Rev. B 71, 155108 (2005).

${ }^{24}$ J. A. Berger, P. Romaniello, R. van Leeuwen, and P. L. de Boeij, Phys. Rev. B 74, 245117 (2006).

${ }^{25}$ R. D'Agosta and G. Vignale, Phys. Rev. Lett. 96, 016405 (2006).

${ }^{26}$ H. O. Wijewardane and C. A. Ullrich, Phys. Rev. Lett. 95, 086401 (2005).

${ }^{27}$ S. Conti and G. Vignale, Phys. Rev. B 60, 7966 (1999).

${ }^{28}$ Z. Qian and G. Vignale, Phys. Rev. B 65, 235121 (2002).

${ }^{29}$ Note that in the case of the combination $\chi(z)=\chi_{1}(z)+\lambda e^{i \omega t} \chi_{2}(z)$, the parity of the state prevents any energy dissipation, i.e., $d E / d t \equiv 0$ for any frequency. 